\title{
Erratum: Thermalization of the Lipkin-Meshkov-Glick model in blackbody radiation [Phys. Rev. A 95, 042107 (2017)]
}

\author{
T. Macrì, M. Ostilli, and C. Presilla \\ (Received 3 September 2017; published 12 October 2017)
}

DOI: 10.1103/PhysRevA.96.049905

In our paper we have erroneously stated that the quantity,

$$
R_{j}=\frac{e^{-\beta E\left(j, m_{z}^{(1)}\right)}}{Z_{j}},
$$

appearing in Eq. (62), attains the limit,

$$
\lim _{N \rightarrow \infty} R_{j}=0,
$$

with an exponential decrease in $N$ in both the paramagnetic and the ferromagnetic regions. However, a proper evaluation of the partition function $Z_{j}$ shows that for $N$ large $R_{j}$ vanishes as $1 / \sqrt{N}$ in the ferromagnetic region and approaches a constant value in the paramagnetic one. The correct result does not affect the conclusions of our paper in any way. In fact, independent of the vanishing speed, $\lim _{N \rightarrow \infty} R_{j}=0$ implies $\lim _{N \rightarrow \infty} \tau^{(P)} \geqslant \lim _{N \rightarrow \infty} \tau^{(Q)}$, a condition effectively used only in the ferromagnetic region.

We also observe that Eqs. (47) and (48) contain some typographical errors, the correct expressions, respectively, being

$$
Z_{j}=e^{\beta \mathcal{J} j(j+1) / N} \sum_{m_{z} \in[-j,-(j-1), \ldots, j]} e^{-\beta m_{z}\left(\mathcal{J} m_{z} / N-\Gamma\right)}=e^{\beta \mathcal{J} j(j+1) / N} \sum_{x \in[-1,-(j-1) / j, \ldots, 1]} e^{-\beta \alpha x N(\mathcal{J} \alpha x-\Gamma)},
$$

and

$$
Z_{j}=\sqrt{\frac{\pi N}{\beta \mathcal{J}}} e^{\beta \mathcal{J} j(j+1) / N} e^{\beta \Gamma^{2} N /(4 \mathcal{J})} .
$$

More importantly, we notice that Eq. (4) (for simplicity we keep only the leading term in $N$ ) holds only for $|\Gamma| N /(2 \mathcal{J})<j$, i.e., in the ferromagnetic region $\Gamma_{c}^{-}<\Gamma<\Gamma_{c}^{+}$, where $\Gamma_{c}^{ \pm}= \pm 2 j \mathcal{J} / N$. Further details on the expression of $Z_{j}$ in the paramagnetic regions $\Gamma<\Gamma_{c}^{-}$and $\Gamma>\Gamma_{c}^{+}$together with an analysis of the ground-state properties of the Lipkin-Meshkov-Glick model will be given elsewhere. 